\title{
Comparação entre medidas de descarga, simetria e transferência de peso em indivíduos com e sem hemiparesia
}

\author{
Comparison between bearing, symmetry, and transfer weight measurements in subjects \\ with or without hemiparesis
}

Emerson Fachin Martins ${ }^{1}$, Paulo Henrique Ferreira de Araujo Barbosa ${ }^{2}$, Lidiane Teles de Menezes², Pedro Henrique Côrtes de Sousa ${ }^{2}$, Abraão Souza Costa ${ }^{2}$

Estudo desenvolvido na

Universidade de Brasília (UnB) Campus UnB Ceilândia - Brasília (DF), Brasil.

${ }^{1}$ Doutor em Psicologia

(Neurociências e Comportamento); Professor Adjunto do curso de Fisioterapia da Faculdade de Ceilândia da UnB - Brasília (DF), Brasil.

2 Estudantes de Fisioterapia (Programa de Iniciação Científica) da Faculdade de Ceilândia da UnB - Brasília (DF), Brasil.

\section{ENDERECO PARA}

\section{CORRESPONDÊNCIA}

\section{Emerson Fachin Martins}

- Universidade de Brasília -

Faculdade de Ceilândia - QNN 14,

Área Especial - Ceilândia Sul - CEP 72220-140 - Brasília (DF), Brasil -

E-mail: efmartins@unb.br

APRESENTAÇÃO

out. 2010

ACEITO PARA PUBLICAÇÃO jun. 2011

FONTE DE FINANCIAMENTO

Fundação de Empreendimentos Científicos e Tecnológicos (FINATEC) - Edital 04/2009; Universidade de Brasília (UnB), Decanato de Pesquisa e Pós-Graduação (DPP/ UnB) - Edital de Auxílio à Pesquisa - 2009, UnBDoc 99456/2009; Universidade de Brasília (UnB), Decanato de Pesquisa e PósGraduação (DPP/UnB), Diretoria de Fomento à Iniciação Científica (DIRIC), Programa de Iniciação Científica 2009-2010 (ProlC/UnB/ CNPq 2009-2010).

\section{CONFLITO DE INTERESSES}

nada a declarar

Parecer de aprovação no Comitê de Ética n 034/2009.
RESUMO: Avaliação da Simetria e Transferência de Peso (ASTP) foi indicada para se fazer associação entre simetria e atividades funcionais nas hemiparesias, apontando simétricos como mais capacitados. Contudo, tais relações não são claras e divergem com evidências que sugerem assimetrias como estratégias funcionais. Assim, objetivou-se verificar se as medidas subjetivamente determinadas pela ASTP concordam com medidas calculas pela descarga de peso entre os pés. Realizou-se estudo observacional do tipo transversal para amostra de sujeitos com hemiparesia $(n=20)$ pareados por idade e gênero a controles $(n=20)$. Os participantes submeteram-se a procedimentos para obtenção de escore determinado pela ASTP e para cálculo da razão de simetria (RS) na descarga de peso entre os pés obtido por meio de duas balanças digitais. Os resultados obtidos pela ASTP identificaram apenas um sujeito com hemiparesia apresentando simetria, dentre os quatro sujeitos identificados pela RS como simétricos. Ainda, a ASTP não diferenciou assimetrias com sobrecarga para o lado afetado e apresentou correlação significativa somente quando os escores foram analisados com os valores de $\mathrm{RS}<1$ (sobrecarga para $\mathrm{O}$ lado não afetado). Conclui-se que a ASTP não foi concordante em identificar sujeitos hemiparéticos com descarga de peso compatível com simetria. Ainda, não identificou sobrecargas para o lado afetado que poderia conduzir a análises equivocadas da associação entre simetria e desempenho funcional.

Descritores: paresia; postura; equilíbrio postural; fisioterapia; acidente cerebral vascular.

ABSTRACT: Assessment of symmetry and weight-transfer (ASWT) was indicated to relate symmetry and functional activity in the hemiparesis, pointing as the most qualified symmetrical. However, such relationships are not clear and disagree with evidences suggesting asymmetries as strategies for functional strategies. Then, it was proposed to verify the measurements subjectively determined by ASWT agree with measurements calculated by weight-bearing distribution for each foot. It was applied observational study with transversal design for sample of subjects with hemiparesis $(n=20)$ matched by age and gender with controls $(n=20)$. Participants were included in procedures toobtain scores by ASWT and to calculate symmetry ratio (SR) in the weight-bearing between feet by digital scales. The results obtained by ASWT indicated only one subject with hemiparesis presenting symmetry among four subjects identified as symmetric by SR. Besides, the ASWT did not differ overweight toward the affected side and presented significant correlation only when the scores were analyzed with values of $\mathrm{SR}<1$ (overweight toward the non-affected side). It was concluded that the ASWT was not agreementto identify weight-bearing distribution compatible with symmetry. Moreover, it did not identify overweight toward the affected side that could to drive to mistaken analysis of the relationship between symmetries and functional performance.

KeYwoRds: paresis; posture; postural balance; physical therapy; stroke. 


\section{INTRODUCÃO}

Descarregar e transferir peso entre os hemicorpos é habilidade útil no processo de reabilitação de sujeitos vítimas de acidente vascular encefálico que adquirem hemiparesias ${ }^{1-7}$. Comumente, em hemiparéticos, a postura em pé é caracterizada por descarga de peso assimetricamente distribuída entre os hemicorpos s,3-6,8-14 $^{\text {. }}$ Apesar de tais sujeitos preferencialmente adotarem um padrão de assimetria que sobrecarrega o lado não afetado, na síndrome de Pusher observa-se postura assimétrica que sobrecarrega o lado afetado $^{15,16}$

Um protocolo sistematizado e elaborado para avaliar a simetria e a transferência de peso pela observação subjetivade hemiplégicos foi descrito e definido pelos autores como Avaliação da Simetria e Transferência de Peso $(\text { ASTP })^{17}$. Apesar de não possuir testes psicométricos e estudos de validação, esta avaliação foi descrita como um instrumento para se inferir a respeito de como assimetrias influenciam nas atividades funcionais de hemiparéticos.

Mesmo sem os devidos procedimentos metodológicos para se utilizar medidas confiáveis e analisar dados com segurança, o estudo que apresentou a ASTP mostrou evidências de que sujeitos mais assimétricos são os mais comprometidos quando ao seu desempenho funcional.

Entretanto, a literatura atual não aponta evidências esclarecedoras que reforçam tal resultado ou de que treinamentos para se adotar descarga de peso mais simetricamente distribuída tenham contribuído para ganhos funcionais ${ }^{12,18-20}$. Ainda, alguns estudos têm demonstrado que assimetrias podem representar uma estratégia para retomada de função em um corpo com resposta motora unilateral ${ }^{19,21}$.

Outra avaliação subjetiva também já foi descrita como alternativa para se verificar assimetrias, contudo tal avaliação utilizou a mão do examinador sob a planta do pé dos sujeitos examinados para determinar a intensidade e o lado em que predominava a descarga de pe$\mathrm{SO}^{22}$, diferindo da avaliação subjetiva feita pela ASTP que utiliza a simples inspeção visual do sujeito para se pontuar itens e determinar a medida por meio de um escore numérico ${ }^{17}$.
É amplamente aceito, há muitos anos na comunidade científica, que as medidas alternativas para avaliação demandam cuidado metodológico e devem ser examinadas por testes psicométricos antes de serem utilizadas ${ }^{23,24}$. Caso contrário, tais medidas estão sujeitas a erros que conduzem a análises equivocadas.

Também de fácil aplicação clínica, medidas mais objetivas e com estudos que já examinaram seu comportamento psicométrico podem ser obtidas por procedimentos utilizando balanças antropométricas digitais e posterior cálculo de índice que informa a distribuição da descarga de peso entre os pés ${ }^{21,23}$.

Frente ao exposto, o presente estudo teve por objetivo verificar se as medidas subjetivamente determinadas pela ASTP concordam com medidas calculas pela descarga de peso entre os pés.

\section{METODOLOGIA}

\section{Sujeitos}

Vinte sujeitos com hemiparesia crônica, com idade variando de 28 a 88 anos, participaram deste estudo (Tabela 1). Procedimentos de amostragem por conveniência foram empregados entre os sujeitos cadastrados nos prontuários do Hospital Regional de Ceilândia. Tais sujeitos não estavam realizando fisioterapia na ocasião do estudo. Para serem incluídos, os participantes deveriam: (1) possuir um período mínimo de seis meses após o acidente vascular encefálico que originou a hemiparesia, (2) ter adquirido sequela do tipo espástica, (3) ser capaz de manter-se em pé por período suficiente para realização dos procedimentos necessários para avaliação pelos instrumentos analisados neste estudo e (4) não fazer uso de dispositivos de apoio. Todos os sujeitos que apresentaram outro tipo de incapacidade para além da hemiparesia foram excluídos. Outros vinte sujeitos sem hemiparesia foram pareados por gênero e idade com todos os sujeitos com hemiparesia para compor o grupo controle (Tabela 1), neste grupo seriam excluídos os sujeitos que: (1) referissem dor durante os procedimentos, (2) apresentassem comprometimentos ortopédicos e/ou neurológicos e (3) utilizassem dispositivos de apoio para marcha. O tamanho final da amostra foi definido com base no cálculo do erro amostra estimado para uma amostra. A inclusão de sujeitos no grupo hemiparesia foi interrompida quando as variáveis (escore e razão de simetria) passaram a apresentar erro amostral estimado menor que 10\% do valor da média. Como o grupo controle foi pareado, o tamanho amostral para este grupo foi determinado pelo tamanho da amostra no grupo hemiparesia.

Todos os participantes assinaram o termo de consentimento livre e esclarecido aprovado pelo Comitê de Ética em Pesquisa da Faculdade de Ciências da Saúde da Universidade de Brasília.

\section{Procedimentos experimentais}

Estudo observacional com delineamento transversal foi usado e as medidas foram tomadas em uma única sessão iniciadas pela ASTP e repetidas pela avaliação mais objetiva da descarga de peso para impedir que o registro objetivo influenciasse na percepção subjetiva do examinador. Durante entrevista, variáveis independentes foram registradas para caracterizar os grupos. As características sociodemográficas incluindo gênero (masculino ou feminino), idade (anos completos) e ocupação antes do dano foram verificadas. Também foram registradas características do acidente vascular encefálico e da sequela hemiparética incluindo cronicidade (meses completos após o evento lesivo), magnitude da incapacidade (hemiplegia ou hemiparesia), território vascular envolvido (artéria cerebral anterior, posterior ou média; artéria basilar, artéria vertebral ou artéria cerebellar) e topografia do comprometimento motor (braquial, crural ou proporcional no hemicorpo). Depois da entrevista, as medidas pelos dois procedimentos definidos para este estudo foram realizadas (variáveis dependentes).

\section{Avaliacão da Simetria e Transferência de Peso (ASTP)}

A avaliação por observação da simetria e transferência de peso foi aplicada conforme descrito por Chagas eTavares ${ }^{17}$. Este instrumento não avalia descarga de peso, mas sim posturas e transferências funcionais que estão organizadas em uma sequência de itens de $\mathrm{A}$ a $\mathrm{H}$ sobre informações obtidas a partir da observação na posição sentado (itens de A a E) e na posição em pé (itens de $\mathrm{F}$ a H). Em cada item observado é registrada a pontuação que varia de 1 a 3 pontos, na posição sentado, e de 1 a 4 
Tabela 1. Características clínicas por sujeito do grupo hemiparesia

\begin{tabular}{lcccccc}
\hline Sujeito & $\begin{array}{c}\text { Idade } \\
\text { (anos) }\end{array}$ & $\begin{array}{c}\text { Cronicidade } \\
\text { (meses) }\end{array}$ & Gênero & Tipo de lesão & Território vascular & Topografia \\
\hline 1 & 57 & 6 & Feminino & Hemorrágico & Artéria vertebral & Braquial \\
2 & 80 & 8 & Feminino & Isquêmico & Artéria cerebral média & Braquial \\
3 & 40 & 11 & Feminino & Isquêmico & Artéria cerebral média & Proporcional \\
4 & 37 & 15 & Masculino & Isquêmico & Artéria cerebral média & Crural \\
5 & 61 & 11 & Masculino & Isquêmico & Artéria cerebral média & Braquial \\
6 & 47 & 10 & Feminino & Isquêmico & Artéria cerebral média & Braquial \\
7 & 52 & 14 & Masculino & Isquêmico & Artéria cerebral média & Crural \\
8 & 55 & 36 & Feminino & Isquêmico & Artéria cerebral média & Braquial \\
9 & 66 & 252 & Masculino & Isquêmico & Artéria cerebral média & Braquial \\
10 & 70 & 24 & Masculino & Isquêmico & Artéria cerebral média & Braquial \\
11 & 44 & 48 & Masculino & Isquêmico & Artéria cerebral média & Braquial \\
12 & 71 & 8 & Masculino & Isquêmico & Artéria cerebral anterior & Crural \\
13 & 59 & 13 & Masculino & Isquêmico & Artéria cerebral média & Braquial \\
14 & 28 & 13 & Feminino & Isquêmico & Artéria cerebral média & Braquial \\
15 & 30 & 22 & Masculino & Isquêmico & Artéria cerebral média & Braquial \\
16 & 71 & 6 & Masculino & Isquêmico & Artéria cerebral média & Crural \\
17 & 62 & 12 & Feminino & Isquêmico & Artéria cerebral média & Proporcional \\
18 & 63 & 9 & Masculino & Hemorrágico & Artéria cerebral posterior & Crural \\
19 & 55 & 45 & Masculino & Isquêmico & Artéria cerebral média & Braquial \\
20 & 36 & 11 & Masculino & Isquêmico & Artéria cerebral média & Braquial \\
\hline
\end{tabular}

pontos, na posição em pé, a depender do julgamento do examinador quanto à mínima e máxima capacidade de simetria e transferências. Quando os itens são somados indicam uma pontuação mínima de 8 e máxima de 27 pontos em uma progressão crescente que indicaria condições mais simetricamente classificadas pelos autores em: ausência de simetria e transferência de peso (8 pontos), capacidade mínima de simetria e transferência de peso (10 a 13 pontos), capacidade moderada de simetria e transferência de peso (14 a 18 pontos), capacidade parcialmente boa de simetria e transferência de peso (19 a 24 pontos), capacidade boa de simetria e transferência de peso (25 a 26 pontos) e capacidade íntegra de simetria e transferência de peso (27 pontos).

\section{Avaliação da descarga de peso}

A simetria na descarga de peso em pé foi avaliada pela razão de distribuição dessa descarga suportada por cada membro inferior entre o lado afetado e o não afetado dos hemiparéticos. A medida do peso suportado em cada lado foi obtida por meio de duas balanças calibradas para uso, com visor digital da marca Plenna ${ }^{\circledR}$ e capacidade máxima de $150 \mathrm{~kg}$.
Os sujeitos eram posicionados descalços, com os pés alinhados, de maneira que o hálux de cada pé ficasse apontado anteriormente, e afastados cerca de $20 \mathrm{~cm}$, sem qualquer forma de suporte auxiliar, com cada um dos membros apoiados separadamente em cada balança.

O visor digital indicava valores inteiros em quilogramas e com uma casa decimal. No momento em que o examinador observava estabilidade na indicação dos valores inteiros apresentados pelo visor em cada balança, a leitura bilateral obtida era registrada. Feito o registro, confirmava-se a equivalência entre o peso corporal total do sujeito e a soma dos valores obtidos nas duas balanças. Caso a soma fosse inferior ou superior a mais de $1 \mathrm{~kg}$ do peso corporal total do sujeito, a leitura era refeita. Os valores obtidos por cada membro foram registrados como valores de descarga no lado afetado e não afetado.

\section{Processamento e análise dos dados}

Para análise, foi calculada a razão de simetria (RS) pela fórmula:

$$
\mathrm{RS}=\frac{\mathrm{a}}{\mathrm{na},}
$$

em que RS refere-se ao valor adimensional da razão de simetria calculada pela divisão do valor de descarga de peso no lado afetado (a) sobre o lado não afetado (na) obtida na avaliação da descarga de peso.

Dessa forma, os valores de $\mathrm{RS}=1$ representariam a simetria absoluta de descarga de peso na posição ortostática. Valores de RS $>1$ representam assimetria de descarga com predomínio para o lado afetado, e valores de $\mathrm{RS}<1$ representam assimetria de descarga com predomínio para o lado não afetado ${ }^{21}$.

Em análise análoga, porém, para os sujeitos do grupo controle, a razão de simetria foi obtida pela divisão do valor de descarga de peso no lado não dominante sobre o lado dominante, sistematizando uma lógica em que a semelhança entre os hemicorpos nos dois grupos se faz pela predominância de uso.

Todas as variáveis foram submetidas ao teste de Kolmogorov-Smirnov para verificar se apresentavam distribuição gaussiana que determinou, para este estudo, o uso de testes paramétricos. Assim, foi assumido que tanto os escores obtidos na ASTP, quanto as razões de simetria registradas por sujeitos, formam variáveis independentes com distribuição gaussiana. 
Teste $t$ de Student para amostras em par foi aplicado para detectar diferenças significativas entre os grupos hemiparesia e controle.

O Intervalo de Confiança de 95\% (IC95\%) da média de escores e razões de simetria obtidos no grupo controle foi usado para estabelecer os limites de simetria, visto que definem um intervalo de valores observados em sujeitos sem hemiparesia. O teste de correlação de Pearson foi usado para se identificar o índice de correlação $\left(r^{2}\right)$ para análises bivariadas. O nível de significância para todas as análises foi estabelecido em $\alpha=0,05$.

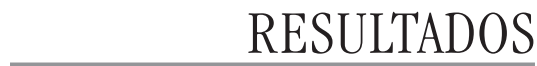

\section{Características dos grupos}

Características sociodemográficas no grupo hemiparesia mostraram uma amostra de sujeitos com 54,20 3,28 (média EPMM) anos de idade com 65\% de homens em ocupações predominantemente representadas pelo grupo de ocupações relacionadas a instalações, manutenção e reparos, conforme definido pela Classificação Padronizada de Ocupações do Departamento de Trabalho dos Estados Unidos $^{25}$. O grupo controle apresentou $55,15 \pm 3,48$ anos de idade com a mesma proporção de gênero observada no grupo hemiparesia, uma vez que os controles foram pareados por idade e gênero com os hemiparéticos. Todos os sujeitos estavam morando na região administrativa de Ceilândia (DF), Brasil.

O grupo hemiparesia apresentou cronicidade de 28,70 $\pm 12,07$ meses, não sendo observados sujeitos classificados como hemiplegia. Dos 20 sujeitos com hemiparesia, 9 (45\%) apresentaram o hemicorpo direito e 11 (55\%) o hemicorpo esquerdo como sendo o lado não afetado, sendo observado prevalência de sujeitos com lesão isquêmica no território de irrigação da artéria cerebral média e predomínio de comprometimento motor braquial no hemicorpo afetado, conforme apresentado na Tabela 1.

\section{Identificação de simetrias e assimetrias}

Para o total de sujeitos, a ASTP pontuou escore médio de 20,85 $\pm 0,80$ para o grupo hemiparesia frente ao escore de $26,20 \pm 0,22$ para o grupo controle, sendo detectada diferença significativa entre os grupos $(p<0,0001)$. Já para a razão de simetria observou-se no grupo hemiparesia valor de 0,86 $\pm 0,08$ frente a um valor de 0,98 $\pm 0,04$ para os controles, não sendo identificada diferença significativa entre os grupos $(p=0,2299)$.

Os limites de simetria definidos pelo IC95\% dos valores obtidos no grupo controle mostraram que a ASTP identificou um único sujeito do grupo

\section{A}

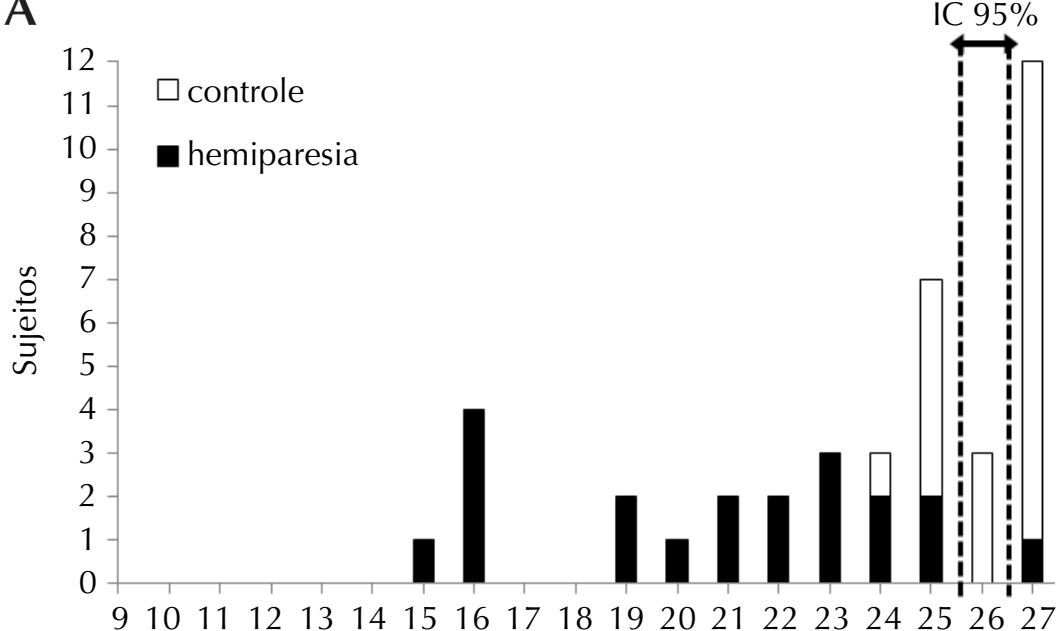

escore

B

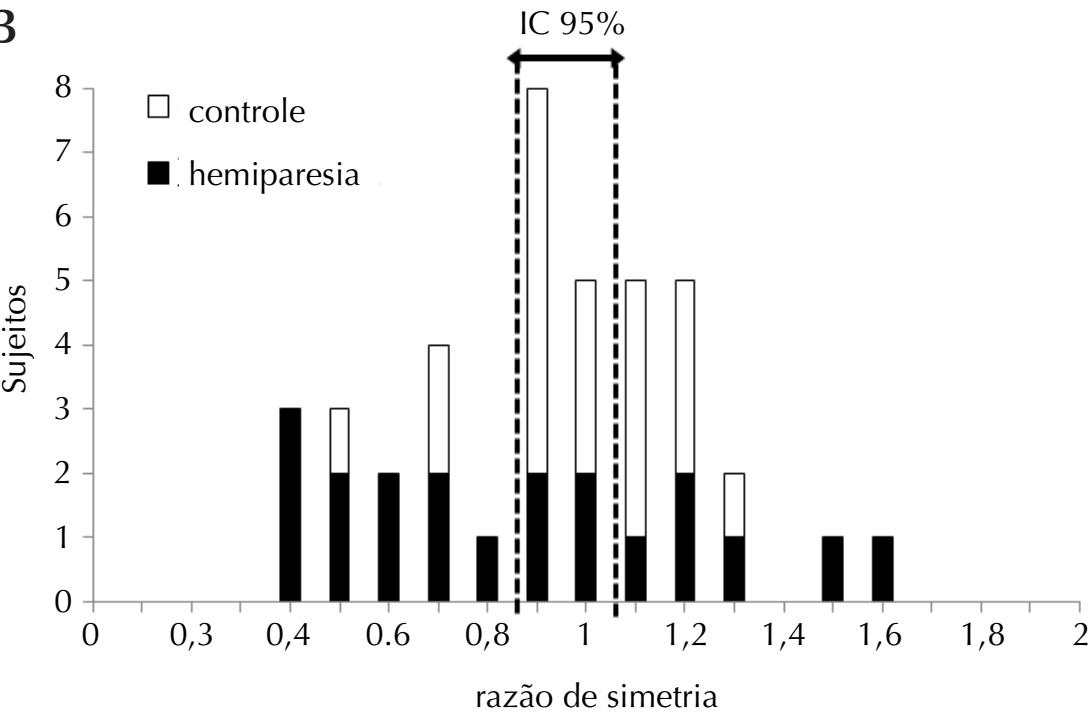

Figura 1. Distribuição de frequência dos sujeitos com (preto) ou sem (branco) hemiparesia definidos pelas classes de escore obtido na avaliação de simetria e transferência de peso (A) e na razão de simetria (B). As linhas pontilhadas delimitam os limites do intervalo de confiança de $95 \%$ calculado para o grupo controle que definiu os valores considerados compatíveis com simetria hemiparesia como simétrico e seis sujeitos do grupo controle como assimétricos, não definindo em qual hemicorpo ocorria a sobrecarga de peso que gerou assimetria (Figura 1A).

Já a razão de simetria identificou 4 sujeitos do grupo hemiparesia como simétricos e 11 sujeitos do grupo como assimétricos; destes carregavam o hemicorpo não predominantemente usado e 3 o hemicorpo predominantemente usado. 


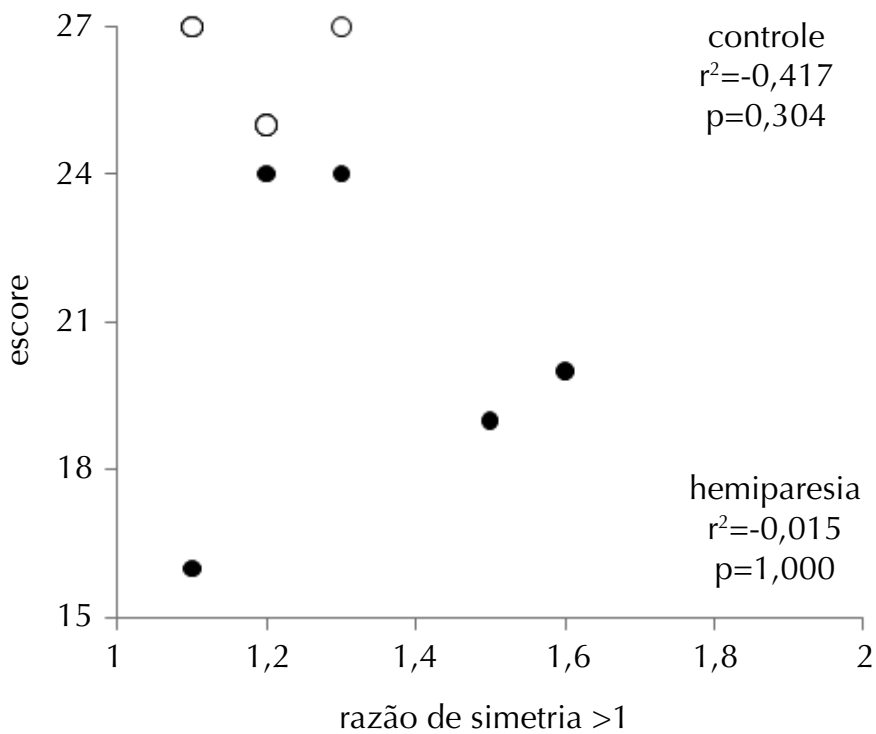

B

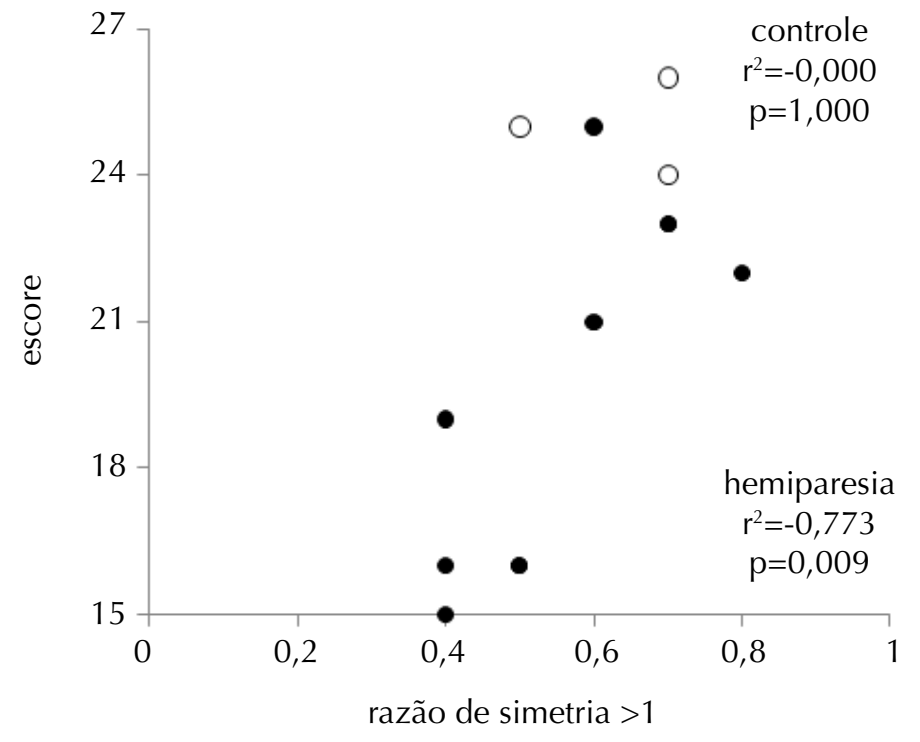

Figura 2. Gráficos de dispersão mostrando a associação dos escores obtidos na avaliação de simetria e transferência de peso (ASTP) com os valores de razão de simetria que indicam sobrecarga para o lado afetado (RS>1) (A) e com os valores de razão de simetria que indicam sobrecarga para o lado não afetado $(R S<1)(B)$ para sujeitos com (pontos pretos) e sem (pontos brancos) hemiparesia. Nos dois gráficos, os índices de correlação $\left(\mathrm{r}^{2}\right)$ e valores de $\mathrm{p}$ para as correlações nos grupos controle e hemiparesia foram indicados à direita. Correlações significativas $(p<0,05)$ foram marcadas com uma estrela

A Figura 1A mostra que a ASTP não diferencia as assimetrias quanto ao hemicorpo sobrecarregado, diferentemente do observado na Figura 2B em que a razão de simetria tornou possível identificar dez sujeitos hemiparéticos assimétricos que sobrecarregavam o hemicorpo predominantemente usado (não afetado, RS $<1$ ) e seis que sobrecarregavam o hemicorpo não predominantemente usado (afetado, RS>1).
Nenhuma correlação significativa foi observada entre os escores obtidos pela ASTP quando testadas com as razões de simetria tanto para o grupo hemiparesia como para o grupo controle (dados não mostrados).

Somente quando o teste de correlação foi aplicado em análises separadas por grupos de predominância de uso (Figura 2 ), é que foi possível se detectar uma correlação forte $(0,773)$ e significativa $(p<0,05)$ entre os escores da ASTP e as razões de simetria no grupo hemiparesia que sobrecarregava o hemicorpo predominantemente usado (Figura 2B), continuando ainda sem correlação significativa entre as variáveis obtidas no grupo controle e mesmo para o grupo hemiparesia cuja sobrecarga ocorria no hemicorpo não predominantemente usado (Figura 2).

DISCUSSÃO

As características etárias dos sujeitos com hemiparesia nesta amostra foram muito similares às observadas em estudo previamente realizado por este grupo que analisou 14 sujeitos portadores de hemiparesia também apresentando heterogeneidade etária (65 \pm 10 anos) e cronicidade variada ( $29 \pm 23$ meses) que não interferiram na análise de correlação ${ }^{21}$.

Concordando com observações epidemiológicas mundiais e nacionais ${ }^{26-28}$, nesta amostra foi observada prevalência de lesão do tipo isquêmica e em território de irrigação da artéria cerebral média (Tabela 1). Ainda, apesar de não ter sido propositalmente controlado no delineamento deste estudo, a prevalência de sujeitos com hemiparesia de comprometimento desproporcionado e com predomínio braquial permitiu a formação de uma amostra em que os membros inferiores, no lado afetado, eram utilizados nas atividades funcionais em bipedestação ${ }^{29}$, talvez determinado pelo critério de inclusão em que os indivíduos deveriam ter a capacidade de permanecer em pé por tempo suficiente para os procedimentos de registro.

Nossos resultados mostraram que a ASTP também pode identificar sujeitos com hemiparesia em posturas compatíveis com simetria e sujeitos sem 
hemiparesia em posturas assimétricas que não foram descritos por Chagas e Tavares ${ }^{17}$, visto que não utilizaram um grupo controle. Assim, para que as evidências apontadas neste estudo apareçam nas análises, seria preciso se delinear procedimentos experimentais que incluíssem um grupo controle que definiria os limites de simetria.

Este estudo também mostrou que o instrumento subjetivo registrou três sujeitos no grupo hemiparesia e cinco sujeitos no grupo controle que foram classificados como simétricos e assimétricos, respectivamente, pela razão de simetria (Figura 1B) e que os mesmos não foram assim classificados pela ASTP (Figura 1A), mostrando que, apesar do instrumento subjetivo também identificar posturas de descarga de peso não tão comuns na população de hemiparéticos e controles, a ASTP não foi acurada o suficiente para concordar com todos os sujeitos classificados pela medida mais objetiva.
Assim como Chagas e Tavares ${ }^{17}$, inúmeros outros estudos ${ }^{1,4,9,11-13,18,21,30-33}$ que analisaram assimetrias posturais não estabeleceram critérios para se classificar simetrias e assimetrias conforme limites estabelecidos em um grupo controle.

O comportamento assimétrico com sobrecarga para o lado afetado, não tão comumente observado, mas presente na população de sujeitos portadores de hemiparesia com síndrome de Pusher ${ }^{15,16}$, não foi reconhecido pela ASTP. Este aspecto pode ter contribuído para a não ocorrência de correlação entre os escores obtidos pela ASTP e os valores de razão de simetria.

Ainda, a correlação significativa das medidas obtidas pelos dois instrumentos observados somente nos sujeitos com hemiparesia cuja postura assimétrica sobrecarregava o lado não afetado (Figura 2B), sugere que o instrumento mais subjetivo tende a concordar com medidas de assimetrias pela maior descarga de peso somente quando a sobrecarga ocorre para o lado não afetado.

\section{CONCLUSÃO}

Conclui-se que a ASTP não é concordante em identificar sujeitos com hemiparesia que apresentam descarga de peso compatível com simetria. Ainda, o instrumento mais subjetivo não identifica sobrecargas para o lado afetado, associando-se apenas com os sujeitos que sobrecarregam o lado não afetado. As conclusões aqui apresentadas sugerem que os usos isolados da avaliação subjetiva sem referência em uma amostra para formar grupo controle e sem um determinante mais objetivo de medida podem conduzir a análises equivocadas da influência das assimetrias observadas nas condições de hemiparesia sobre indicadores de desempenho funcional.

\section{REFERÊNCIAS}

1. Chen $\mathrm{CH}$, Lin $\mathrm{KH}$, Lu TW, Chai HM, Chen HL, Tang PF, et al. Immediate effect of lateral-wedged insole on stance and ambulation after stroke. Am J Phys Med Rehabil. 2010;89(1):48-55.

2. Mercer VS, Freburger JK, Chang SH, Purser JL. Measurement of paretic-lower-extremity loading and weight transfer after stroke. Phys Ther. 2009;89(7):653-64.

3. Barra J, Oujamaa L, Chauvineau V, Rougier P, Perennou D. Asymmetric standing posture after stroke is related to a biased egocentric coordinate system. Neurology. 2009;72(18):1582-7.

4. Genthon N, Rougier P, Gissot AS, Froger J, Pelissier J, Perennou D. Contribution of each lower limb to upright standing in stroke patients. Stroke. 2008;39(6):1793-9.

5. van Asseldonk EHF, Buurke JH, Bloem BR, Renzenbrink G), Nene AV, van der Helm FCT, et al. Disentangling the contribution of the paretic and non-paretic ankle to balance control in stroke patients. Exp Neurol. 2006;201(2):441-51.

6. Perennou D. Weight bearing asymmetry in standing hemiparetic patients. J Neurol Neurosurg Psychiatry. 2005;76(5):621.

7. Rodriguez GM, Aruin AS. The effect of shoe wedges and lifts on symmetry of stance and weight bearing in hemiparetic individuals. Arch Phys Med Rehabil. 2002;83(4):478-82.
8. Patterson KK, Gage WH, Brooks D, Black SE, Mcllroy WE. Evaluation of gait symmetry after stroke: a comparison of current methods and recommendations for standardization. Gait Posture. 2010;31(2):241-6.

9. Rougier PR, Genthon N. Dynamical assessment of weight-bearing asymmetry during upright quiet stance in humans. Gait Posture. 2009;29(3):437-43.

10. Nardone A, Godi M, Grasso M, Guglielmetti S, Schieppati M. Stabilometry is a predictor of gait performance in chronic hemiparetic stroke patients. Gait Posture. 2009;30(1):5-10.

11. Myklebust JB, Lovett EG, Myklebust BM, Reynolds N, Milkowski L, Prieto TE. Two-dimensional coherence for measurement of asymmetry in postural steadiness. Gait Posture. 2009;29(1):1-5.

12. Van Peppen RPS, Kortsmit M, Lindeman E, Kwakkel G. Effects of visual feedback therapy on postural control in bilateral standing after stroke: a systematic review. J Rehabil Med. 2006;38(1):3-9.

13. Marigold DS, Eng JJ. The relationship of asymmetric weight-bearing with postural sway and visual reliance in stroke. Gait Posture. 2006;23(2):249-55.

14. McCombe Waller S, Whitall J. Hand dominance and side of stroke affect rehabilitation in chronic stroke. Clin Rehabil. 2005;19(5):544-51. 


\section{Referências (cont.)}

15. Babyar SR, Peterson MG, Bohannon R, Perennou D, Reding M. Clinical examination tools for lateropulsion or pusher syndrome following stroke: a systematic review of the literature. Clin Rehabil. 2009;23(7):639-50.

16. Johannsen L, Broetz D, Karnath HO. Leg orientation as a clinical sign for pusher syndrome. BMC Neurol. 2006;6:30.

17. Chagas EF, Tavares M. A simetria e transferência de peso do hemiplégico: relação dessa condição com o desempenho de suas atividades funcionais. Rev Fisiot Univ São Paulo 2001;8(1):40-50.

18. de Haart M, Geurts AC, Huidekoper SC, Fasotti L, van Limbeek J. Recovery of standing balance in postacute stroke patients: a rehabilitation cohort study. Arch Phys Med Rehabil. 2004;85(6):886-95.

19. Roerdink M, Geurts AC, de Haart M, Beek PJ. On the relative contribution of the paretic leg to the control of posture after stroke. Neurorehabil Neural Repair. 2009;23(3):267-74.

20. Teixeira-Salmela LF, Lima RCM, Lima LAO, Morais SG, Goulart F. Assimetria e desempenho funcional em hemiplégicos crônicos antes e após programa de treinamento em academia. Rev Bras Fisioter. 2005;9(2):227-33.

21. Pereira LC, Botelho AC, Martins EF. Relationships between body symmetry during weight-bearing and functional reach among chronic hemiparetic patients. Rev Bras Fisioter. 2010;14(3):229-66.

22. Gray FB, Gray C, McClanahan JW. Assessing the accuracy of partial weight-bearing instruction. Am J Orthop (Belle Mead NJ). 1998;27(8):558-60.

23. Hurkmans HL, Bussmann JB, Benda E, Verhaar JA, Stam HJ. Techniques for measuring weight bearing during standing and walking. Clin Biomech (Bristol, Avon). 2003;18(7):576-89.

24. Yelnik A, Bonan I. Clinical tools for assessing balance disorders. Neurophysiol Clin. 2008;38(6):439-45.
25. US-DL. United States Department of Labor - Standard Occupational Classification - Major Groups. 2010 [cited 2010 Aug 1st]; Available from: http://www.bls.gov/soc/ major_groups.htm

26. Schinwelski M, Slawek J. Prevalence of spasticity following stroke and its impact on quality of life with emphasis on disability in activities of daily living. Systematic review. Neurol Neurochir Pol. 2010;44(4):404-11.

27. Cavalcante TF, Moreira RP, de Araujo TL, Lopes MVD. Demographic factors and risk indicators of stroke: comparison between inhabitants of Fortaleza municipal district and the national profile. Rev Latino-Am Enfermagem. 2010;18(4):703-8.

28. Hosseini AA, Sobhani-Rad D, Ghandehari K, Benamer HTS. Frequency and clinical patterns of stroke in Iran - Systematic and critical review. BMC Neurology. 2010;10:72.

29. Stephenson JL, De Serres SJ, Lamontagne A. The effect of arm movements on the lower limb during gait after a stroke. Gait Posture. 2010;31(1):109-15.

30. Tung FL, Yang YR, Lee CC, Wang RY. Balance outcomes after additional sit-to-stand training in subjects with stroke: a randomized controlled trial. Clin Rehabil. 2010;24(6):533-42.

31. Campanini I, Merlo A. Reliabilty, smallest real difference and concurrent validity of indices computed from GRF components in gait of stroke patients. Gait Posture. 2009;30(2):127-31.

32. Lomaglio MJ, Eng JJ. Muscle strength and weight-bearing symmetry relate to sit-to-stand performance in individuals with stroke. Gait Posture. 2005;22(2):126-31.

33. Goldie PA, Matyas TA, Evans OM, Galea M, Bach TM. Maximum voluntary weight-bearing by the affected and unaffected legs in standing following stroke. Clin Biomech (Bristol, Avon). 1996;11(6):333-42. 